\title{
Development and Research of Bypass Device to Improve DC Grid Performance
}

\author{
Jongman Kim ${ }^{1}$, Seokhoon Hong ${ }^{2}$, Beobseong Song ${ }^{2}$ and Yong-sung Choi ${ }^{3}$ \\ 1. Jeonnam State University, Jeonnam 57337, Republic of Korea \\ 2. TEF Company Limited, Jeonnam 57903, Republic of Korea \\ 3. Dept. of Electrical Engineering, Dongshin University, Jeonnam 58245, Republic of Korea
}

\begin{abstract}
Among the factors determining the performance of the new renewable energy system, the processing part of the untreated DC power is a very important element. In fact, the area where the loss of the new renewable energy system is greatest is also the DC transmission and distribution technology. It is critical to prevent this loss and to improve the performance of the system, and analyze the performance of the resulting through the application of the resulting algorithms. In this thesis, we are discussing ways to calibrate the error in the PV distribution system within the solar power generation system using the Bypass Line Configuration Algorithm. I would like to note that if the operation of the Bypass Line device generates electrical abnormalities in the system's circuits, it can recover the performance of the system, thereby improving performance.
\end{abstract}

Key words: DC grid, bypass diode, DC transmission.

\section{Introduction}

Photovoltaic systems vary in performance depending on the change in environmental factors, even though installation sites, methods, power conditions, forms, etc. are the same. In order to achieve the full performance of the solar power system, it is necessary to quantify the performance characteristics including installation and installation conditions of high-performance products, and the overall performance characteristics, such as the performance estimation, and the loss of performance [3]. Improved reliability is also needed to improve the performance of the solar power generation system. All solar power stations consist of a string circuit separated by a minimum generator for each generation unit, and are generally not reliable for the over-current isolation devices inserted in each circuit. In order to improve the performance of the system problem, such as shutdown systems due to low stability, we intend to develop a

Corresponding author: Jongman Kim, Ph.D., professor, research fields: renewable energy, electrical engineering. joint control technology, power quality and supply stabilization system for the application of large scale systems.

\section{Automatic Power Transfer System Control Management Unit}

\subsection{Proposed String Configuration System}

In the case of the currently distributed systems, the contact with the load on the load cell module that is connection board is located in the center of the colony, and the power line of all the modules in the vicinity is taken in and processed. It has aspects that violate the criteria for determining whether power is normal. This can be found in terms of convenient management and risk management, which can minimize problems, which are common requirements for solar power plant operators as well as clients.

First, it should establish a system which ensures convenient construction, effective management, and minimizes risk by improving electrical stability and providing parts that meet management standards. In addition, the configuration of the solar control system 
must be ensured in order to maintain electrical protection of the solar power system and to maintain the smooth electric power generation performance and electrical efficiency. In this paper, we will deal with the automatic switching system, its monitoring system and communication method for safety management when abnormal voltage is generated.

\subsection{Automatic Power Conversion System Control Management}

In monitoring and control system of DC power of solar power plant, system for transmission and distribution is constructed by using elements such as blocking diodes to extend system stability. In addition, the development of control devices using the power sensor module and the monitoring system using the remote communication technology are also integrated into the solar power management system in Fig. 1.

The techniques and components required for the development of stable DC power processing and transmission and distribution to be proposed in this paper are as follows.

(1) Constructing integrated system by combining developed element technologies;

(2) Configuration of current processing unit with DC collector by unit capacity;

(3) Composition of communication network through wireless communication module built in DC collecting device;

(4) Comprehensive data collection and processing.

In this system, the current/voltage value is measured by the sensor and input to the MCU (micro controller unit), and the comparison operation determines whether to shut off or open the circuit.

Fig. 2 is a block diagram showing the method of the automatic power conversion system.

\subsection{Real Time Control Algorithm}

The development of a method for increasing the stability of the power connection point of the photovoltaic system can improve the performance of the system. This system is used as a power electronic component for installation, and can be applied in the field without a procedure such as specification or certification.

In order to facilitate the configuration and management of the communication lines of the power line and the sensor module in the per-channel power processing system, a system of $30-40 \mathrm{~kW}$ unit is usually constructed. Fig. 3 shows the generic configuration of a real-time sequence for PV system control.

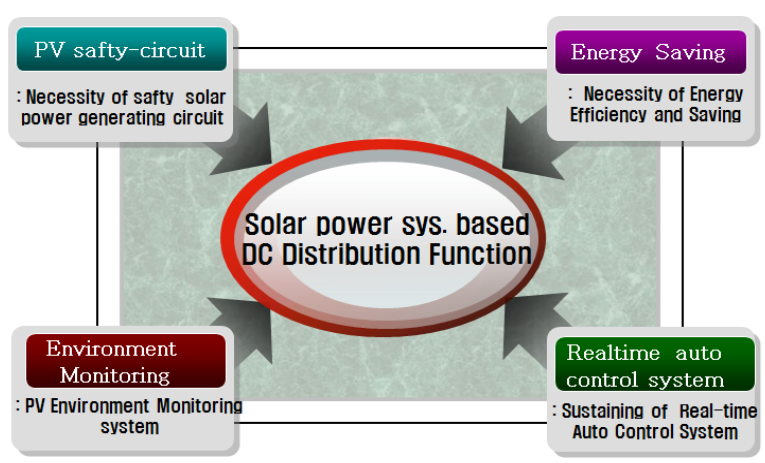

Fig. 1 The final development system of proposed solar power DC distribution system.

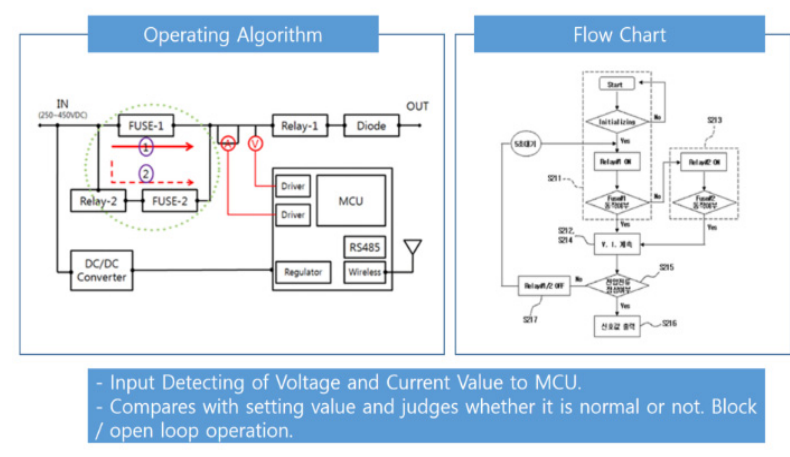

Fig. 2 Automatic power conversion system block diagram.

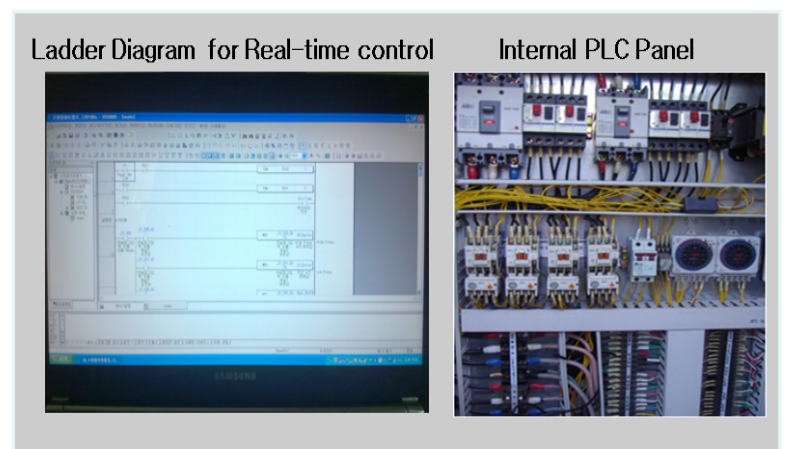

Fig. 3 Generic model of real-time sequence logic for PV system control. 
Unlike the system in Fig. 3, which is generally used, the characteristics of the proposed system are as follows.

The automatic power switching system has improved the stability of the system by constructing a circuit for improving the stability of the photovoltaic system. The basic line operation connecting the fuse-1 to the relay-1 to the first lane is provided as a line that is normally operated. When the power abnormality is physically detected or is detected by an abnormality of the parameter, it is switched to Relay- $2 \rightarrow$ Fuse- 2 line through the operation of the automatic power switching operation circuit to correct the operation clearance according to instantaneous power change [1].

Fig. 4 shows the operating circuit in the steady state, and Fig. 5 shows the operating state of the automatic power switching system when the fault occurs.

In order to improve system performance, it is necessary to integrate the monitoring system configuration technology. Power monitoring and monitoring services are not widely available in low-capacity photovoltaic power generation facilities. This is because a large investment cost is required to construct a power management supplementary service

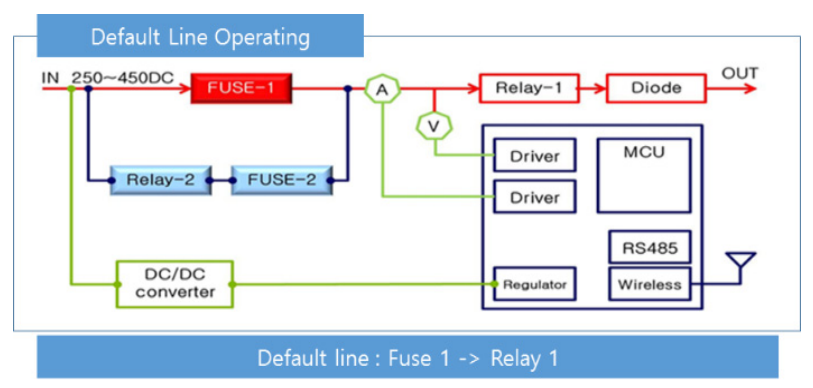

Fig. 4 Basic line operation of bypass circuit.

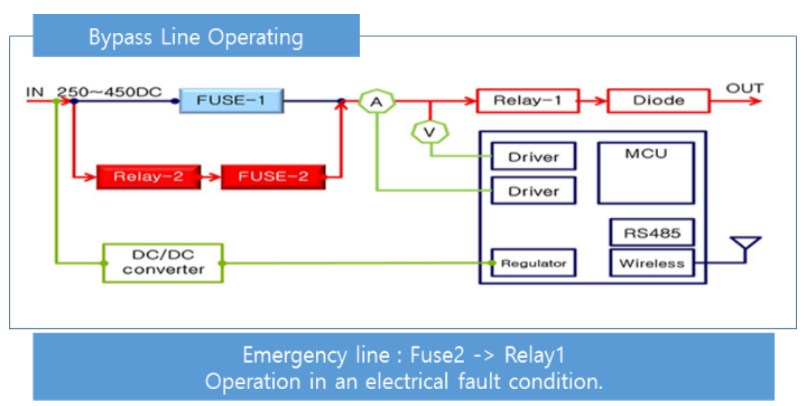

Fig. 5 Bypass line operation circuit for increasing photovoltaic power safety. system for a low-capacity photovoltaic power generation facility.

The application of this system is technically and economically worthy of alleviating the problem of the installation cost that has been a problem in the meantime [2].

In the case of a communication system, when using a wired communication system such as RS-422 or RS-485, communication stability is good and signal fading is small, which is suitable for use in a power plant. In case of using wireless communication, it is advantageous to use $400 \mathrm{MHz}$ RF method rather than WIFI or Bluetooth method for improving communication quality. Fig. 6 is the example for wireless communication system linking topology.

(1) In the case of parallel processing of data with a small amount of data (several Kbytes), there are a mode bus method and a serial data processing method which are methods used for processing a plurality of devices. However, when such a communication method causes a problem in synchronization, it seriously damages received data.

(2) The communication system designed to solve the problem distinguishes between short-distance communication and long-distance communication, and the communication load is reduced by completely separating the data acquisition unit from the data transmission/reception unit.

(3) In the case of short range communication, it acquires the analog information of the unit module and internally $\mathrm{A} / \mathrm{D}$, and transfers it to the long distance network. The data transmitted by the local area communication device having the plurality of unique IDs are inserted into the protocol of the long distance communication device and transmitted to the server.

\subsection{Circuit Configuration and Design}

The technic circuits and design data are required for stable DC power processing and transmission to be proposed in this paper are as follows.

Fig. 7 shows the operating circuits for SMPS (Switching 


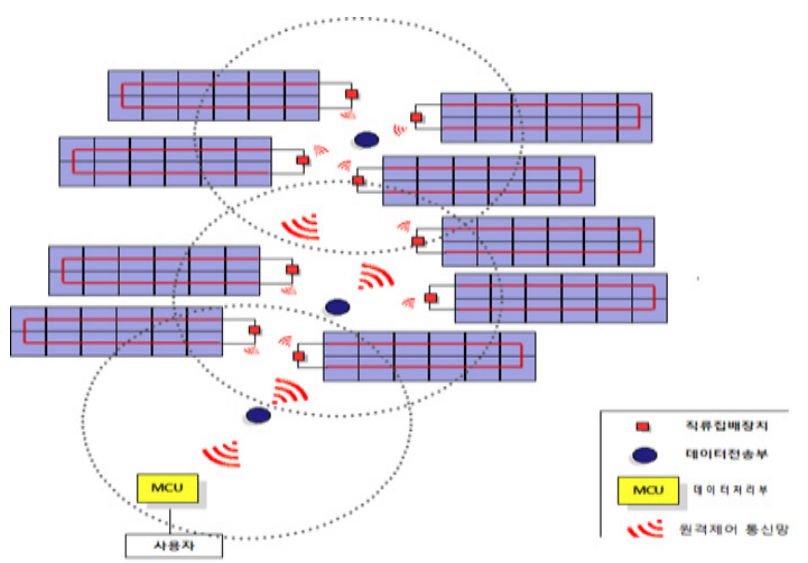

Fig. 6 DC photovoltaic power generation system.

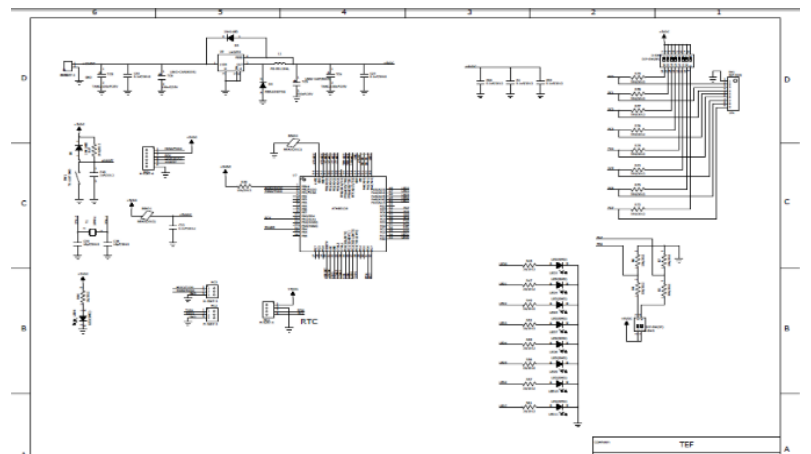

Fig. 7 Configuration of SMPS Power, MCU, Switch, LED.

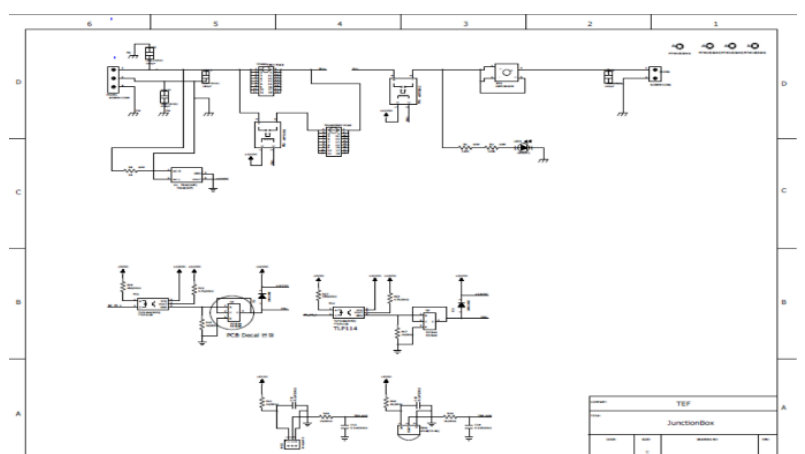

Fig. 8 Configuration of the operating circuits for Fuses and Diodes.

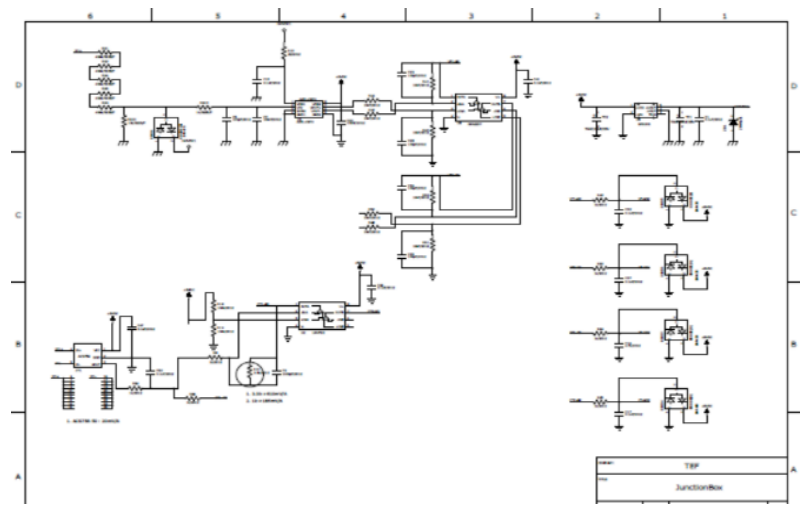

Fig. 9 Configuration of voltage-current sensing circuits.
Mode Power Supply) Power, MCU, Switch, LED, and Fig. 8 shows the operating circuits for Fuses and Diodes. Fig. 9 shows configuration of voltage-current sensing circuits, and Fig. 10 shows configuration of wireless communication part. Fig. 11 shows configuration of artwork layout.

\section{Conclusion}

\subsection{System Performance Test and Analysis}

The circuit configuration and characteristics test of this system are as follows.

\subsubsection{Stabilization Circuit of the Pickup Circuit}

It is a protection circuit against spark voltage generated when PV open voltage or PV abnormality is set to 2 3 times of open voltage.

\subsubsection{Breaking Current Measurement Test}

The blocking/automatic power switching function is activated at the proper current, and it is possible to realize the stable bypass function. Results of breaking current measurement test are in Fig.12 as follows.

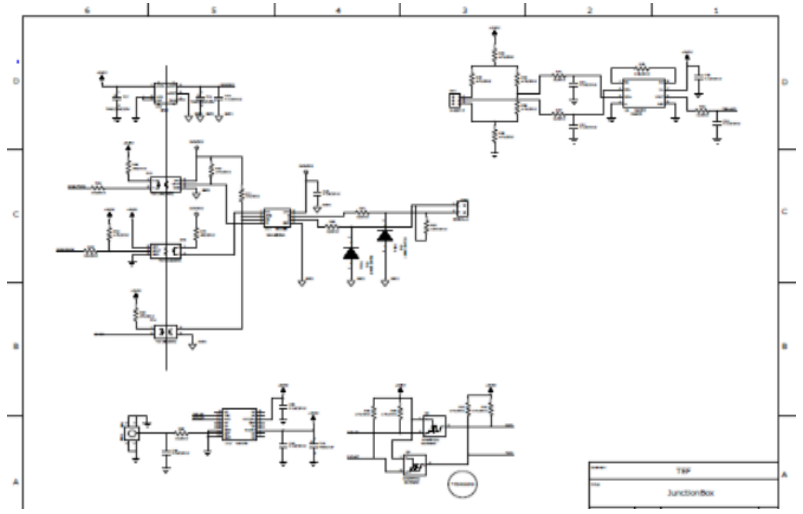

Fig. 10 Configuration of wireless communication part.

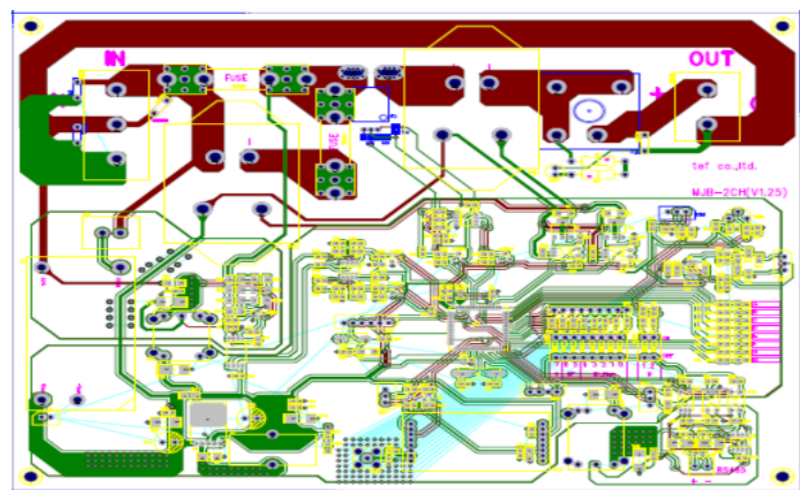

Fig. 11 Configuration of artwork layout. 


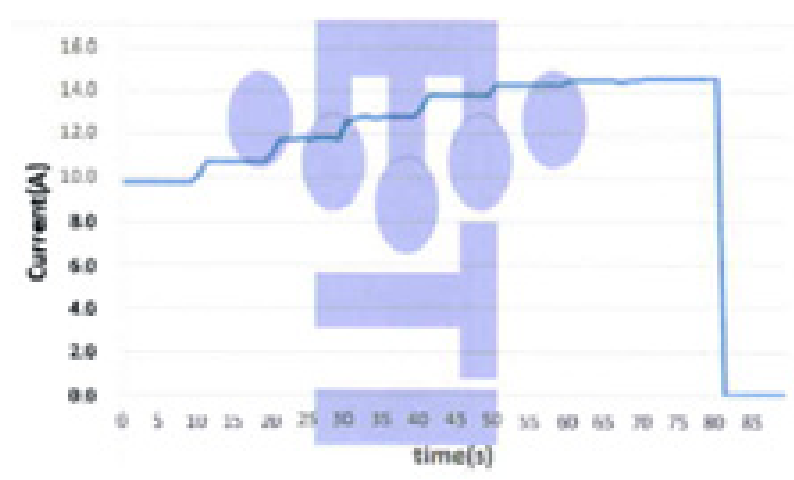

Fig. 12 Results of breaking current measurement tests.

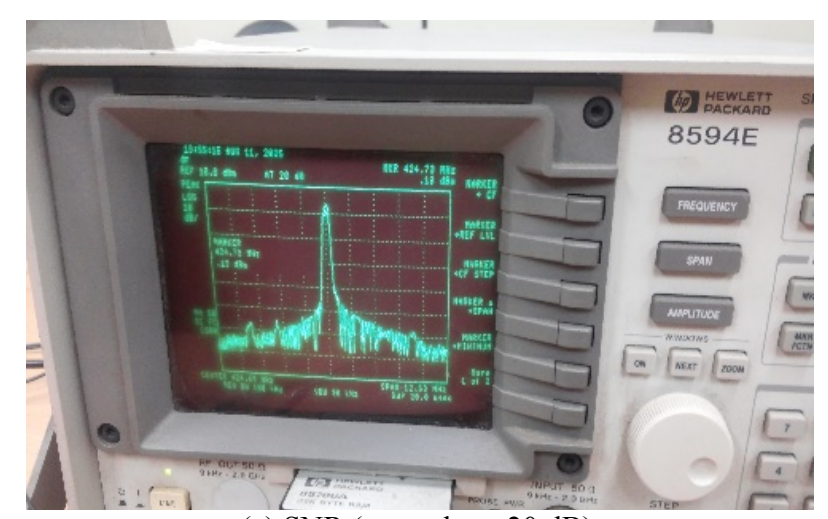

(a) SNR (more than $-20 \overline{\mathrm{dB}}$ )

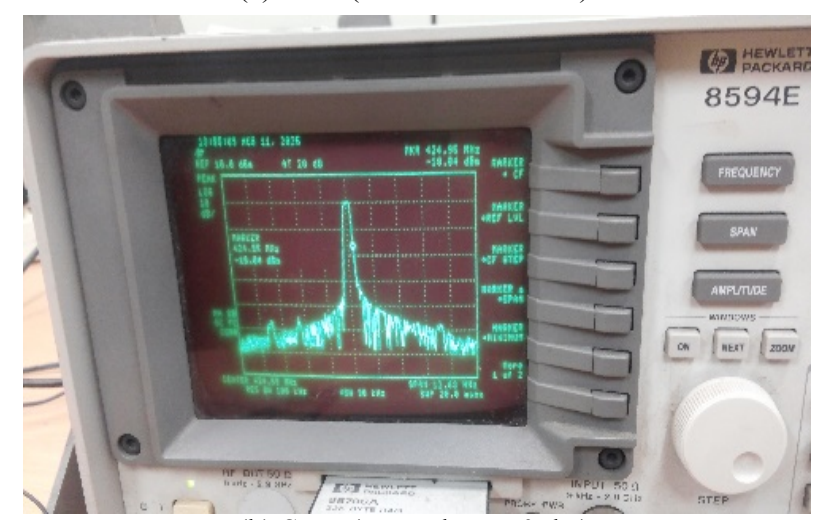

(b) SNR (more than $-50 \mathrm{~dB}$ )

Fig. 13 Results of system performance analysis.

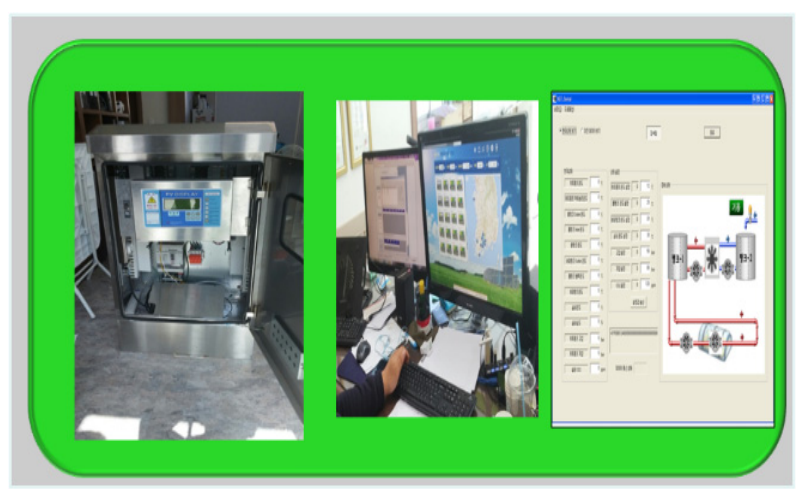

Fig. 14 System test and monitoring system configuration.

\subsubsection{System Performance Analysis}

(1) The SNR (signal-to-noise ratio) of the system is more than $-20 \mathrm{~dB}$ and shows excellent performance in Fig. 13.

(2) The SNR of the noise region was found at more than $-50 \mathrm{~dB}$, and the transmission quality compared to the noise band of the signal was found to be no problem to apply to the field.

\subsection{Configuration of Management System}

A real-time monitoring system was constructed for monitoring and managing the system in Fig. 14.

\subsection{Conclusion}

In case of solar system voltage fuse failure, it is possible to monitor the overvoltage and overcurrent through the function of changing to auxiliary fuse through power relay, so that the automatic shutoff function can be confirmed when an abnormality occurs in the line.

The system has a characteristic of determining whether the line is basically opened or closed and actively judging whether the line is momentarily maintained or blocked by sensing a change in the state of the input power.

In addition, it is confirmed that real-time management of characteristics with channel compensation and emergency shutdown function is possible by constructing a real-time monitoring system for control for monitoring and managing the system.

We propose a wireless communication method of communication method used in PV power plant and through this, performance verification is accomplished. This system is an important research/development for the maintenance of the photovoltaic system and it is considered that the value of the additional research will be high in the future.

\section{Acknowledgement}

This work was supported by the Industry-University Fusion Center, under the establishing of Development 
of DC Power Distributed Device for DC grid Power Infrastructure.

\section{References}

[1] Kim, S. T., Park, C. H., Kang, G. H., Waithiru, C. K., Lawrence, H. K., Ahn, G. J., et al. 2007. "Operation Characteristics of Bypass Diode for PV Module.” Journal of KIEEME 21 (1): 12.

[2] Gratzel, M. 2000. "Perspectives for Dye-Sensitized Nonocrystalline Solar Cells.” Prog. Photovoltaics Res. Appl. 8: 171.

[3] Kaminski, A., Vandelle, B., Fave, A., Boyeaux, J. P., Nam, L. Q., Monna, R., et al. 2002. "Aluminium BSF in Silicon Solar Cells.” Solar Energy Material \& Solar Cells 72 (1-4): 373-9. 